\title{
Comparison of Otoacoustic Emissions Within Gecko Subfamilies: Morphological Implications for Auditory Function in Lizards
}

\author{
Christopher Bergevin \\ Department of Mathematics, University of Arizona, Tucson, AZ 85705, USA \\ Present address: Department of Otolaryngology/Head and Neck Surgery, Columbia University, 630 W. 168th St. P\&S 11-452, New York, \\ NY 10032, USA
}

Received: 27 September 2010; Accepted: 16 November 2010; Online publication: 7 December 2010

\begin{abstract}
Otoacoustic emissions (OAEs) are sounds emitted by the ear and provide a non-invasive probe into mechanisms underlying peripheral auditory transduction. This study focuses upon a comparison of emission properties in two phylogenetically similar pairs of gecko: Gekko gecko and Hemidactylus turcicus and Eublepharis macularius and Coleonyx variegatus. Each pair consists of two closely related species within the same subfamily, with quantitatively known morphological properties at the level of the auditory sensory organ (basilar papilla) in the inner ear. Essentially, the comparison boils down to an issue of size: how does overall body size, as well as the innerear dimensions (e.g., papilla length and number of hair cells), affect peripheral auditory function as inferred from OAEs? Estimates of frequency selectivity derived from stimulus-frequency emissions (emissions evoked by a single low-level tone) indicate that tuning is broader in the species with fewer hair cells/shorter papilla. Furthermore, emissions extend outwards to higher frequencies (for similar body temperatures) in the species with the smaller body size/narrower interaural spacing. This observation suggests the smaller species have relatively improved high-frequency sensitivity, possibly related to vocalizations and/or aiding azimuthal sound localization. For one species (Eublepharis), emissions were also examined in both juveniles and adults. Qualitatively similar emis-
\end{abstract}

Correspondence to: Christopher Bergevin · Department of Otolaryngology/Head and Neck Surgery, Columbia University, 630 W. 168th St. P\&S 11-452, New York, NY 10032, USA. email: dolemitecb@gmail.com sion properties in both suggests that inner-ear function is adult like soon after hatching and that external body size (e.g., middle-ear dimensions and interaural spacing) has a relatively small impact upon emission properties within a species.

Keywords: otoacoustic emission, gecko hearing, tuning, SFOAE delay, age differences

Abbreviations: ABR - Auditory brainstem response; ANF - Auditory nerve fiber; BM - Basilar membrane; CAP - Compound action potential; dB SPL - Decibels re-sound-pressure level; DPOAE - Distortion product otoacoustic emission; ME-Middle ear; OAE Otoacoustic emission; $Q$ - Quality factor; SFOAE Stimulus-frequency otoacoustic emission; SOAE Spontaneous otoacoustic emission; SVL - Snout-vent length; TM - Tectorial membrane

\section{INTRODUCTION}

There is a remarkable amount of morphological diversity of the lizard inner ear (e.g., Miller (1973a); Wever (1978)). For example, the arrangement of sensory cells (or hair cells) along the basilar papilla and the structure of the tectorial membrane (TM; a thin gelatinous matrix that couples directly to the hair cells) can vary dramatically across species. This diversity has in turn been exploited in order to better understand the function of auditory transduction in the vertebrate ear (Manley 2000, 2002). In addition to 
testing evolutionary conjectures, the simple fact of existence of such inner-ear morphological diversity in the Lacertilia raises questions about how the mechanics of hearing differs across various lizard groups. Previous studies have made systematic comparisons across a wide variety of lizard species, focusing on the structure and function of the TM (Manley 1997; Bergevin et al. 2010b). However, other features such as the total number of hair cells also varied significantly amongst the species compared. Such differences can potentially introduce confounding factors for interpretation, suggesting a desire to make comparisons across lizard species in a more phylogenetically matched context.

Despite the diversity of the ear across various lizard families, there can be relative similarity within closely related taxonomic groupings. While properties such as papilla length and number of hair cells can vary within a given subfamily, the general morphological structure of the inner ear appears qualitatively homogeneous (Wever 1978; Miller 1973a). For example, the structure of the TM (continuous, discretized, or even absent) and orientation of the hair cell bundles appear fairly uniform within lizard families. These similarities provides an anchor point for testing hypotheses with regard to how various morphological properties affect function. More specifically, qualitative similarity within a given subfamily allows for known quantitative differences (e.g., hair cell count, overall body size) to be exploited to address questions about subsequent functional consequences. As an example, two previous studies (Manley et al. 1996; Manley 2009) looked at differences within (and across) lizard families to better understand the processes that give rise to the spontaneous generation of sound from the ear, known as otoacoustic emissions (OAEs).

Otoacoustic emissions are sounds emitted from the healthy ear and measurable non-invasively using a sensitive microphone. These emissions can arise either spontaneously (as noted above) or via an evoking stimulus and are generally considered a byproduct of an underlying amplification process at work in the inner ear to improve detection of lowlevel signals (Probst et al. 1991). While much attention has focused on understanding OAE generation mechanisms in the mammalian cochlea, emissions are a fairly universal feature in most types of ears, including non-mammals such as birds, amphibians and non-vertebrates. For reasons presently unclear, lizards appear to be particularly robust emitters (e.g., Manley (2006); Bergevin et al. (2008)). One key difference relative to mammals is that there is evidence indicating that lizards lack a traveling wave propagating along the length of their basilar membrane (BM) (Peake and Ling 1980; Manley et al. 1988). As BM waves play a significant role in many classes of theoretical models for mammalian $\mathrm{OAE}$ generation, the lack of such in lizards can help simplify interpretation of their OAEs. Thus, given the relative simplicity of their inner-ear anatomy and physiology, lizards serve as a good animal model for elucidating OAE generation mechanisms and in turn, processes underlying auditory transduction (Manley 2000, 2002).

Stimulus-frequency otoacoustic emissions (SFOAEs), evoked via single tones, have been proposed as a means to non-invasively probe the frequency selectivity of the inner ear (Shera et al. 2002; Moleti and Sisto 2003; Shera and Guinan 2003). Recent reports focusing on the lizard ear have lent support, both theoretically (Bergevin and Shera 2010) and empirically (Bergevin et al. 2010b). A central approach of these studies is the use of the steady-state emission phase-gradient delay (i.e., the slope of the response phase with respect to frequency as the stimulus tone is swept). The basic idea is that SFOAE delays are indicative of auditory filter build-up times in the inner ear, and thus in turn reflect the associated sharpness of tuning. It has been previously shown that tuning estimates stemming from spontaneous emissions (SOAEs) and low-level SFOAEs correlate well to neurophysiological tuning measures in lizards (Manley et al. 1996; Bergevin and Shera 2010) as well as mammals (Shera and Guinan 2003; Shera et al. 2010). However, it has been suggested that other delay sources (e.g., middle-ear transmission) that are not directly associated with tuning could be significant and thus affect interpretation of SFOAE delays and their relation to estimates of tuning (e.g., see Shera et al. (2010)).

\section{BASIS FOR A COMPARATIVE STUDY}

The goal of the present study is to make comparisons across lizard species in a phylogenetically matched fashion, thereby allowing for a more direct connection between features observed in OAE properties and the underlying morphology. We focus here exclusively on lizard species within the family Gekkonidae. The gecko auditory system appears special amongst lizards (Miller 1973b; Manley 2010): complex papilla structure and TM morphology, "reversed" tonotopic map (Manley et al. 1999) and robust emissions (Manley et al. 1996; Stewart and Hudspeth 2000; Bergevin et al. 2008). Additionally, Gekkota is the only lizard infraorder known to possess elasticized vocal cords (Russell et al. 2000) and display spectrally rich vocalizations (Marcinelli 1977; Weber and Werner 1977). Furthermore, there is a significant body of auditory physiological work that has been reported on geckos, such as age-related changes in auditory sensitivity (e.g., Werner et al. (1998)), 
dependence upon body temperature (Campbell 1969; Werner 1976), and various comparative descriptions (e.g., Wever et al. (1964); Werner et al. (1998); Ganeshina and Vorobyev (2009); Brittain-Powell et al. (2010)). Auditory nerve fiber (ANF) responses reported for Gekko gecko (Eatock et al. 1982; SamsDodd and Capranica 1994; Manley et al. 1999) are relatively sharply tuned, similar to cat (Liberman 1990) though with characteristic frequencies limited to below $5 \mathrm{kHz}$. Auditory brainstem responses (ABR) have also been reported for Gekko (BrittainPowell et al. 2010). Additionally, a recent report indicated that the gecko family Pygopodidae has exceptional high-frequency sensitivity (Manley and Kraus 2010), apparently correlated to the frequency content of their vocalizations (Weber and Werner 1977). Lastly, theoretical models for OAE generation in gecko have been described (Bergevin and Shera 2010; Gelfand et al. 2010). Taken together, geckos thus make an ideal group for a systematic study of how morphological features relate to OAE generation.

We consider two pairings (see Table 1) here such that each pair comes from a particular gecko subfamily: Gekkoninae-Gekko gecko and Hemidactylus turcicus and Eublepharinae-Eublepharis macularius and Coleonyx variegatus. These two subfamilies are fairly different based upon external appearance and behavior. For example, species from Gekkoninae tend to be highly vocal, have fused eyelids, and possess toe pads enabling them to readily scale vertical surfaces. Conversely, Eublepharinae members have more limited vocalizations, unfused eyelids, and clawed feet. However, both Gekkoninae and Eublepharinae appear to have similar overall inner ear morphology (Wever 1978). Most notably, the overall papillar structure is divided into three distinct regions (Miller 1973b; Wever 1978; Manley et al. 1996; Chiappe et al. 2007; Bergevin et al. 2008): (1) a basal section covered by a continuous TM with unidirectionally oriented bundles and sensitive to frequencies below $\sim 1 \mathrm{kHz}$, (2) an apical/abneural section covered by a discretized TM (sallets) with bidirectionally oriented bundles and sensitive to frequencies above $\sim 1 \mathrm{kHz}$, and (3) an apical/neural section covered by a continuous TM with bidirectionally oriented bundles and apparently lacking afferent innervation, unlike the other two regions.

To first order, the main difference between the two species within a given pair is size, both in terms of overall body proportions as well as inner ear dimensions. Tables 1 and 2 indicate that for each pair, the smaller species has a shorter papilla with fewer hair cells, in addition to smaller physical dimensions (e.g., body mass and snout-vent length (SVL)). Although not explicitly quantified in this study, the smaller species in each pair also has narrower interaural spacing between tympanic membranes. This spacing has implications for azimuthal sound localization and may thus be related to the range of audition (i.e., frequencies the lizard is most sensitive to). Furthermore, a structure called the fundus (situated between the basilar membrane and basilar papilla) appears relatively thinner in the smaller species (i.e., less papillar mass) (Wever 1978). Lastly, the relative proportions of the papilla's three sub-divisions could also be different. For example, the apical segment (comprising regions 2 and 3 as noted above) appears disproportionately larger in the smaller species of the pair (Wever 1978).

In order to distinguish between effects due to body size versus inner ear properties, a fifth group is also examined: juvenile Eublepharis. Based upon overall body size/appearance, juvenile Eublepharis and adult Coleonyx are similar (see Table 1). There is evidence that lizard inner ear morphology is relatively independent of age (Miller 1985; Gehr and Werner 2005), indicating that the juvenile Eublepharis inner ear morphology is adult-like. Thus, juvenile Eublepharis has a body size similar to adult Coleonyx, but inner ear dimensions similar to adult Eublepharis. A previous report (Werner et al. 1998) indicated that auditory sensitivity in two gecko species (including Eublepharis)

TABLE 1

Overview of quantitative morphological differences across species

\begin{tabular}{lccccc}
\hline & \multicolumn{3}{c}{ Morphological parameters } \\
\cline { 2 - 6 } Species & Subfamily & Mass $(\mathrm{g})$ & SVL $(\mathrm{cm})$ & Papilla (mm) & No. of HCs \\
\hline Gekko gecko & Gk & $\sim 34-66$ & $\sim 10-15$ & $1.7-2$ & $1,625-2,200$ \\
Hemidactylus turcicus & Gk & $2.5-3.6$ & $4.9-5$ & 0.9 & $650-790$ \\
Eublepharis macularius & Eb & $\sim 24-60$ & $\sim 11.1-12.4$ & $1.2-1.4$ & $900-970$ \\
E. macularius (juvenile) & Eb & $3.3-4.6$ & $5.1-5.9$ & $1.2-1.4$ & $900-970$ \\
Coleonyx variegatus & Eb & $2.5-3.2$ & $5.1-5.7$ & 0.8 & $435-515$ \\
\hline
\end{tabular}

Acronyms for gecko subfamilies are as follows: Gekkoninae (Gk), Eublepharinae (Eb). Values for mass and snout-vent length (SVL) are ranges from the animals used in the present study. Values for papilla length and hair cell (HC) count are from the following references: Miller (1973a, b), Wever (1978), Köppl and Authier (1995), Gehr and Werner (2005). Values cited in the table indicate the range reported from these studies. All values are from adults, except for Eublepharis where values from juveniles are also given (1-2 weeks post-hatching). Various lines of evidence indicate juvenile Eublepharis inner ear dimensions are similar to adults (Miller 1985; Gehr and Werner 2005). The SVL is the same measure as the rostrum-anus length (RA) as reported in previous studies (e.g., Gehr and Werner (2005)) 
TABLE 2

Capsule summary of comparison for adults

\begin{tabular}{lll}
\hline & L-Gekkoninae & II-Eublepharinae \\
\hline Large & Gekko (Tokay gecko) & Eublepharis (Leopard gecko) \\
Small & Hemidactylus (House gecko) & Coleonyx (Banded gecko) \\
\hline
\end{tabular}

Each family is grouped into a column, indicating the two species within a given comparison pairing. Each row indicates the corresponding relative "size" category for that species within the pair: Small—shorter papilla w/ fewer hair cells, smaller body size, narrower interaural spacing, Large-longer papilla w/ more hair cells, larger body size, wider interaural spacing. Note that juvenile Eublepharis is not included within this classification scheme, as it would fall into both the top (due to the adult-like inner ear) and bottom rows (due to having similar body dimensions to Coleonyx). Common names have also been noted

changes with age, adults being more sensitive than juveniles by $5-15 \mathrm{~dB}$ (this difference varying with frequency and across species). Further study indicated that some degree of this difference can be attributed to middle-ear properties (Werner et al. 1998, 2002, 2008). Taken together, the juvenile Eublepharis data should help to decouple variations due to overall body size from those due to differences in the inner ears of Eublepharis and Coleonyx. Overall, the present comparative approach is similar to the triads used by Werner et al. (2002) when examining age/size-related effects.

The morphological differences are outlined in Table 1 and suggest several function-related questions that will be addressed in the present study:

1. How do low-level SFOAE properties (e.g., magnitudes, phase-gradient delays) vary with size within a given pair? Across subfamilies?

2. Does the frequency range of detectable OAEs change with size?

3. Are SFOAE properties similar/different between adults and juveniles within a given species?

4. In what ways are the answers to the above questions related to functional and morphological aspects (e.g., interaural spacing, number of hair cells, vocalizations)?

\section{METHODS}

All measurements reported in this study were obtained using the same stimulus paradigms, acquisition codes, and OAE probe for all species/individuals (Bergevin et al. 2008, 2010b). A desktop computer housed a 24-bit soundcard (Lynx TWO-A, Lynx Studio Technology), whose synchronous root mean square input/output was controlled using a custom data-acquisition system. A sample rate of $44.1 \mathrm{kHz}$ was used to transduce signals to/from an Etymotic ER-10C probe containing a microphone and two earphones. The microphone response was amplified by $40 \mathrm{~dB}$ and high-pass filtered with a cutoff frequency of $0.41 \mathrm{kHz}$ to minimize the effects of noise. The OAE probe was coupled to the external ear using a short tube attached to the foam tip and sealed to the head using vaseline or silicone grease. This ensured a tight (closed) acoustic coupling and minimized lowfrequency losses. The probe tip was $\sim 0.5-1.25 \mathrm{~cm}$ from the tympanic membrane. The probe earphones were calibrated in situ by presenting flat-spectrum, randomphase noise. By computing the ratio of response to that of the output signal, the frequency response and associated delays could be determined. Calibrations were verified repeatedly throughout the experiment. All stimulus frequencies were quantized such that an integral number of cycles were contained within the sampling window. To evoke the SFOAEs, a low probe level $\left(L_{\mathrm{p}}=20 \mathrm{~dB}\right.$ SPL) was chosen for several reasons as discussed in a previous report (Bergevin et al. 2010b).

The range of stimulus frequencies $\left(f_{\mathrm{p}}\right)$ employed was typically $0.4-8 \mathrm{kHz}$. The stimulus and emission frequencies are one and the same for SFOAEs. A twotone suppression paradigm was employed to extract the SFOAE (Shera and Guinan 1999; Bergevin et al. 2008). The suppressor parameters were: $f_{\mathrm{s}}=f_{\mathrm{p}}+40 \mathrm{~Hz}, L_{\mathrm{s}}=L_{\mathrm{p}}+15 \mathrm{~dB}$ (where $f_{\mathrm{s}}$ and $L_{\mathrm{s}}$ are the suppressor frequency and level, respectively). A total of 35 waveforms (8192 sample window) were averaged, excluding any flagged by an artifact-rejection paradigm (Shera and Guinan 1999). A period of $\sim 20 \mathrm{~ms}$ was allowed before the start of the sample window, allowing for the associated response to reach steady-state. Frequency step-size during sweeps was small enough to avoid ambiguity during the phase unwrapping. Delays associated with the measurement system were determined and subtracted out. The noise floor was defined as the average sound-pressure level centered about (but excluding) the frequency of interest. It was quantified via averaging the magnitudes of the \pm 3 bins in the fast Fourier transform (FFT) of the response.

The phase-gradient delay, expressed either in time $\left(\tau_{\mathrm{OAE}}\right)$ or number of stimulus periods $\left(N_{\mathrm{SFOAE}}\right)$, is obtained from the derivative of the phase function with respect to probe frequency $\left(f_{\mathrm{p}}\right)$. It is given by

$$
N_{\mathrm{SFOAE}}=f_{\mathrm{p}} \tau_{\mathrm{OAE}}=-\frac{f_{\mathrm{p}}}{2 \pi} \frac{\partial \phi_{\mathrm{OAE}}}{\partial f_{\mathrm{p}}}
$$

where $\phi_{\mathrm{OAE}}$ is the emission phase (in radians) and $f_{\mathrm{p}}$ is in hertz.

Delays $\left(\tau_{\mathrm{OAE}}\right)$ were computed from individual (unwrapped) phase responses using centered dif- 
ferences (Shera and Guinan 2003). As shown in Figures 1, 2, and 3, delay trends were computed across individuals of a given species via a locally weighted regression (loess) (Cleveland 1993) (smoothing parameter $\alpha \approx 0.3$, polynomial of degree one, robust fit). A small amount of dithering was used for the horizontal ordinate of each data point (since delays were typically measured at the same frequencies across individuals), improving the trends ability to capture "local" behavior. To further reduce the effects of outliers at the end points, only $N_{\mathrm{SFOAE}}$ values whose corresponding magnitude (as well as the magnitude of its neighbors) was at least $10 \mathrm{~dB}$ above the noise floor were included in the fits. This criterion
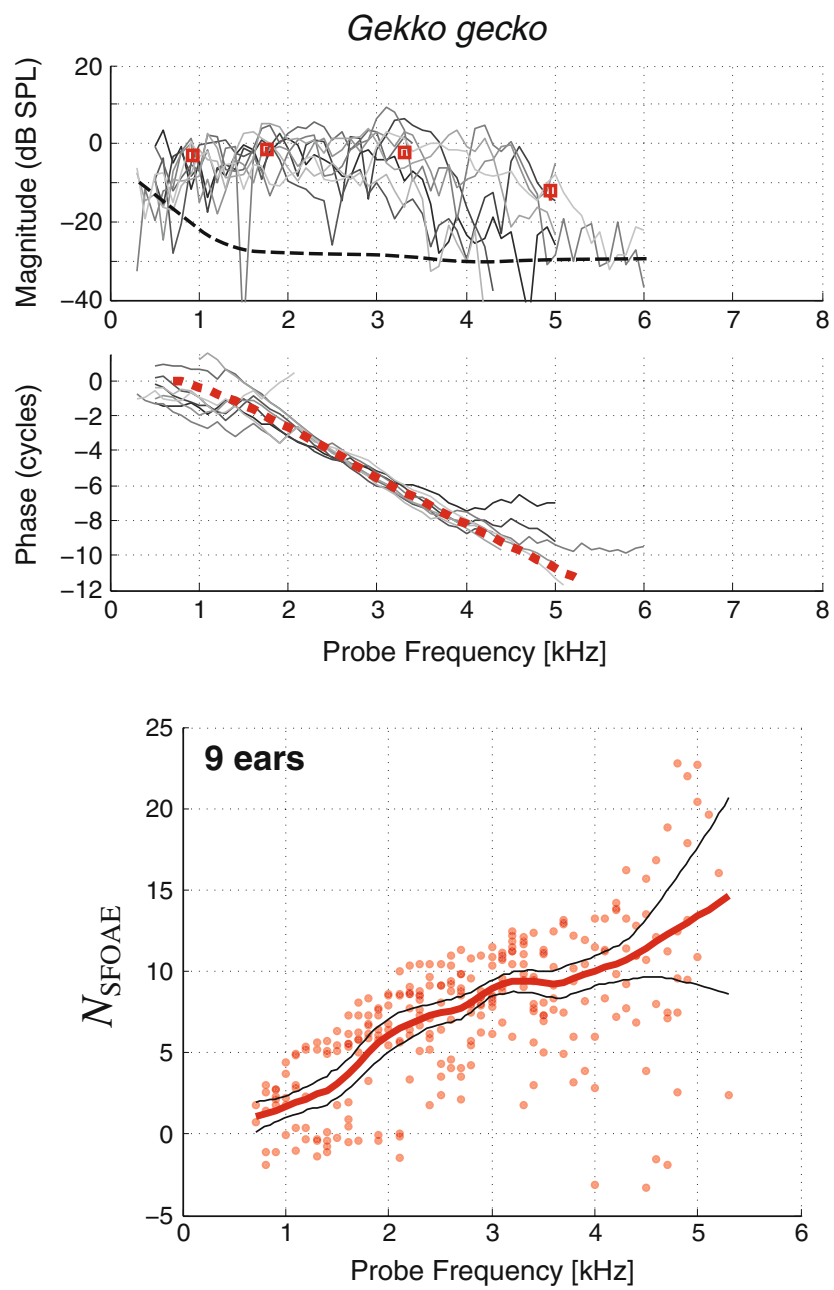

FIG. 1. Compiled adult SFOAE data for both Gekko and Hemidactylus, evoked using a stimulus level of $L_{\mathrm{p}}=20 \mathrm{~dB}$ SPL. Top plots show magnitude and phase (error bars of individual curves excluded for clarity, different individuals are shown via different shading intensities). Average magnitudes (and associated standard error) within octave-wide bins are indicated via the red square symbols (See "Methods"). Approximate noise floor is indicated via the dashed black line. Dashed red line for the phase indicates the $N_{\mathrm{SFOAE}}$ loess trend integrated with respect to frequency, thereby providing the "average" phase response for visual comparison. Some phase curves have been offset vertically for provided a useful measure of the transition from detectable emission to noise floor, indicated by the Cutoff frequency listed in Table 3 .

Uncertainty in the loess trends was computed via bootstrapping: By randomly re-sampling the data (using the same total number of points) and recomputing the trend, the corresponding standard deviation across fits could be computed. Such computations provided the corresponding $95 \%$ confidence intervals, as plotted for $N_{\mathrm{SFOAE}}$ in Figures 1, 2, 3 , and 4. Additional details about the nature of $N_{\mathrm{SFOAE}}$ (and the associated loess fits) can be found in previous reports (Shera and Guinan 2003; Bergevin et al. 2010b). It is worth noting that SFOAE delays estimated from phase-gradients appear well corre-
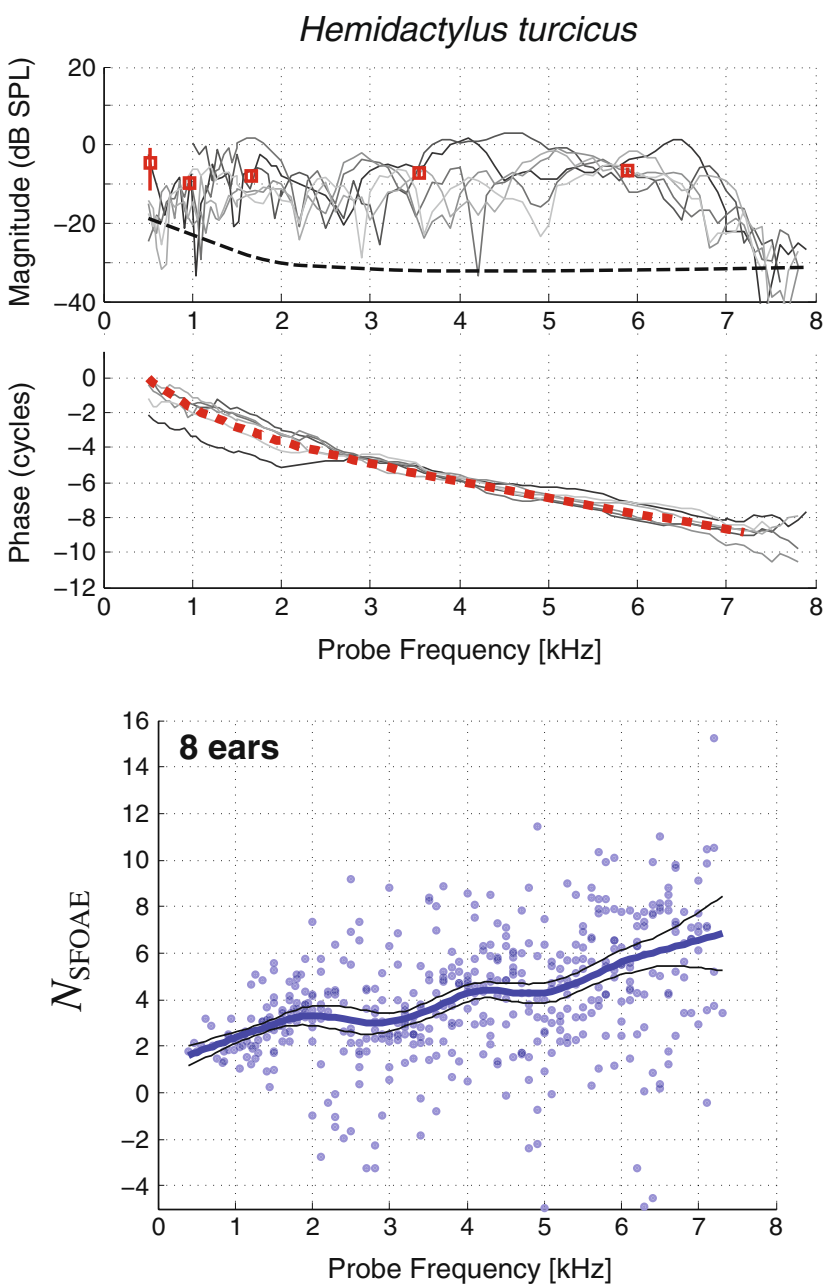

clarity. Bottom plot $\left(\mathrm{N}_{\mathrm{SFOAE}}\right)$ indicates the phase-gradient delay (in cycles) as computed from the phase response. Only points whose magnitude was at least $10 \mathrm{~dB}$ above the noise floor were included. Solid line for $N_{\text {SFOAE }}$ plot is a locally weighted regression (loess) while solid black lines indicate the associated 95\% confidence interval (see "Methods"). The total number of unique ears is indicated in the upper left corner of the $N_{\text {SFOAE }}$ plots. All data were obtained with lizards at a steady-state body temperature of $\sim 32-33^{\circ} \mathrm{C}$ via a regulated heating pad. Other stimulus parameters used: $L_{\mathrm{s}}=35 \mathrm{~dB}$ $\mathrm{SPL}, f_{\mathrm{s}}=f_{\mathrm{p}}+40 \mathrm{~Hz}$. 

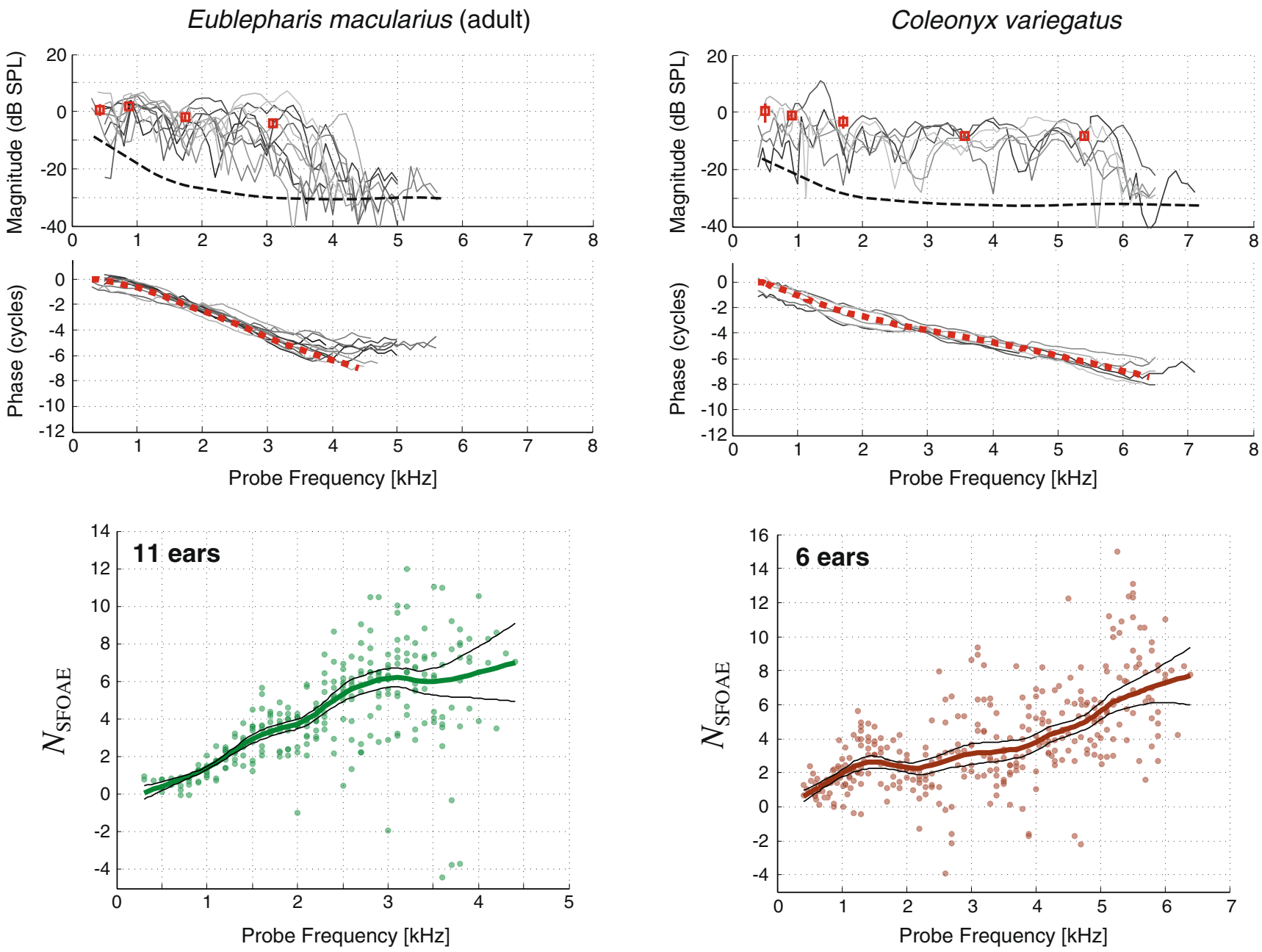

FIG. 2. Similar to the previous figure, except data are shown for adult Eublepharis and Coleonyx.

lated to delays measured in the time domain for both frogs and humans (Meenderink and Narins 2006; Sisto et al. 2007), as expected theoretically. Also, different methods for estimating tuning from SFOAEs can lead to different results (e.g., Lineton and Wildgoose (2009)). However, we use the same method uniformly for all individuals in the present study.

Data from Hemidactylus, Coleonyx, and juvenile Eublepharis were obtained from experiments at the University of Arizona with approval from the Institutional Animal Care and Use Committee. Experiments were performed during the months of June-July. Hemidactylus and Coleonyx (native to southern Arizona) were wild-caught by hand. They were held for approximately 1-2 days, after which the experiments were performed. After a 1-2-day recovery period, lizards were released back at the site of capture. Juvenile Eublepharis were hatched from eggs stemming from two healthy adult females and one male. The hatchlings were housed in glass terraria with a 9-h light cycle and fed small meal worms and crickets starting 1 week after hatching. The juvenile Eublepharis individuals were studied between 7 and 16 days posthatching. The reported data for Hemidactylus come from eight ears from four unique individuals, Coleonyx — six ears from three individuals and juvenile Eublepharis—six ears from four individuals. Data from Gekko and adult Eublepharis come from a previous study (Bergevin et al. 2008), using the same paradigm as outlined here. For all species in this study, OAE data were collected from both males and females and from both ears in a given individual; the results as presented here do not distinguish between sex nor between data collected from left versus right ears (see "Results" for discussion).

All lizards used in this study appeared healthy and active. Prior to each experiment, an animal was anesthetized via a $22-25 \mathrm{mg} / \mathrm{kg}$ Nembutal intraperitoneal injection to prevent movement. These doses were effective for approximately 1 to $3 \mathrm{~h}$, though in several instances, some individuals required an additional half dose. All animals recovered completely within a few hours after the experiment. During the experiments, lizards were placed in a noise-attenuating chamber. Body temperature was kept constant by 

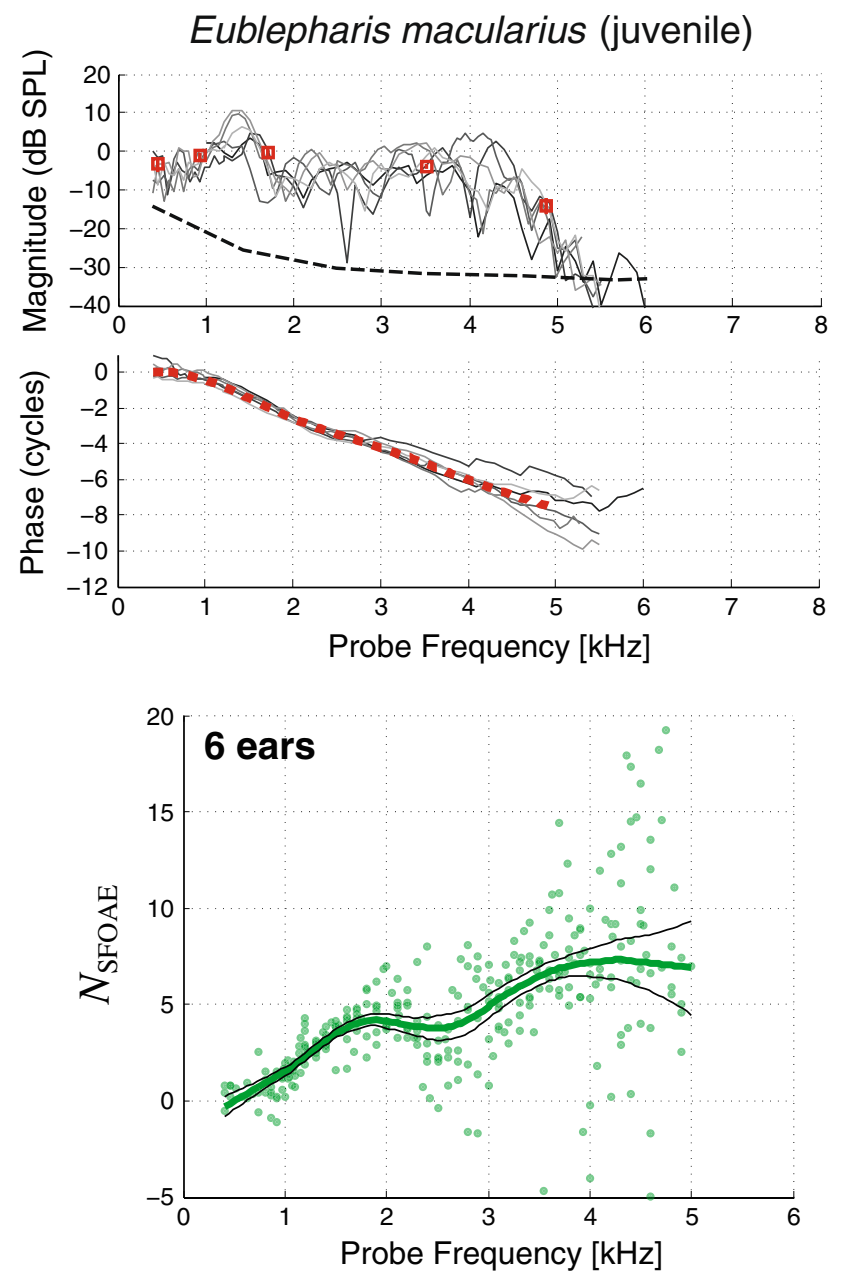

FIG. 3. Similar to the previous figures, except data are shown for juvenile Eublepharis.

the use of a regulated heating blanket (Harvard Apparatus) and monitored using a calibrated thermocouple placed in the mouth (propping it open) or in the leg pit for cases where the lizard spit out the thermometer. Body temperature was kept in the range of approximately $32-33^{\circ} \mathrm{C}$ (verified via a quick-reading cloacal thermometer). Preliminary data indicate SFOAE phase-gradient delays appear rela- tively insensitive to depth of anesthetic state or temperature (Bergevin et al. 2010a).

In Figures 1, 2, and 3, average magnitudes were computed as follows. All values at least $10 \mathrm{~dB}$ above the noise floor were placed into octave bins starting at $0.3 \mathrm{kHz}$ and extending up to $9.6 \mathrm{kHz}$ (as such, there were instances where the corresponding bin was empty because no points met the required threshold criterion). Averages (along with the standard error of the mean) were computed in pascals and converted back to dB SPL. The corresponding horizontal ordinate as shown in Figures 1, 2, and 3 indicates the geometric mean frequency of all points within the given bin.

Although not systematically reported here, some results stemming from SOAE measurements are noted. For SOAEs, 60 waveforms (32768 sample window, $\mathrm{SR}=44.1 \mathrm{kHz}$ ) were acquired and the FFT magnitudes spectrally averaged, either with or without a suppressor tone present. SOAE activity could be readily distinguished in that it was both temperature-dependent as well as entrained to a nearby external tone (Köppl and Manley 1993b; Manley et al. 1996).

\section{RESULTS}

Results for SFOAEs evoked using a low-level stimulus $\left(L_{\mathrm{p}}=20 \mathrm{~dB}\right.$ SPL $)$ are shown in phylogenetically matched pairings in Figures 1 and 2. For a given species in each pair, the top plots show the magnitude and phase of the emission while the bottom plot shows the associated phase-gradient delay expressed in number of stimulus cycles ( $N_{\mathrm{SFOAE}}$; see "Methods"). The $N_{\text {SFOAE }}$ plots also include a locally weighted regression (loess) to indicate the trend. That trend was subsequently numerically integrated to produce the corresponding phase curve, as shown via the dashed line for the phase plot. Also included are the average magnitudes (and associated standard error of the mean) for magnitudes lumped into one-octave

TABLE 3

The level column indicates the approximate range of the average magnitudes (see Fig. 1 caption) over the frequency range $\sim 1-4 \mathrm{kHz}$, where emissions were most prominent in all species. Cutoff indicates the approximate frequency at which emission magnitudes started to become indistinguishable from the noise floor, as inferred from the loess trends given the $10 \mathrm{~dB}$ criterion (see "Methods"). The final column gives the approximate range of time delays $\left(\tau_{\mathrm{OAE}}\right)$ for frequencies above $1.5 \mathrm{kHz}$. These delays can also be inferred from Figure 4 by dividing $N_{\mathrm{SFOAE}}$ trend by the emission frequency $($ Eq. 1$)$. Stimulus parameters used: $L_{\mathrm{p}}=20 \mathrm{~dB} \mathrm{SPL}, L_{\mathrm{s}}=$ $35 \mathrm{~dB} \mathrm{SPL}$, and $f_{\mathrm{s}}=f_{\mathrm{p}}+40 \mathrm{~Hz}$ 


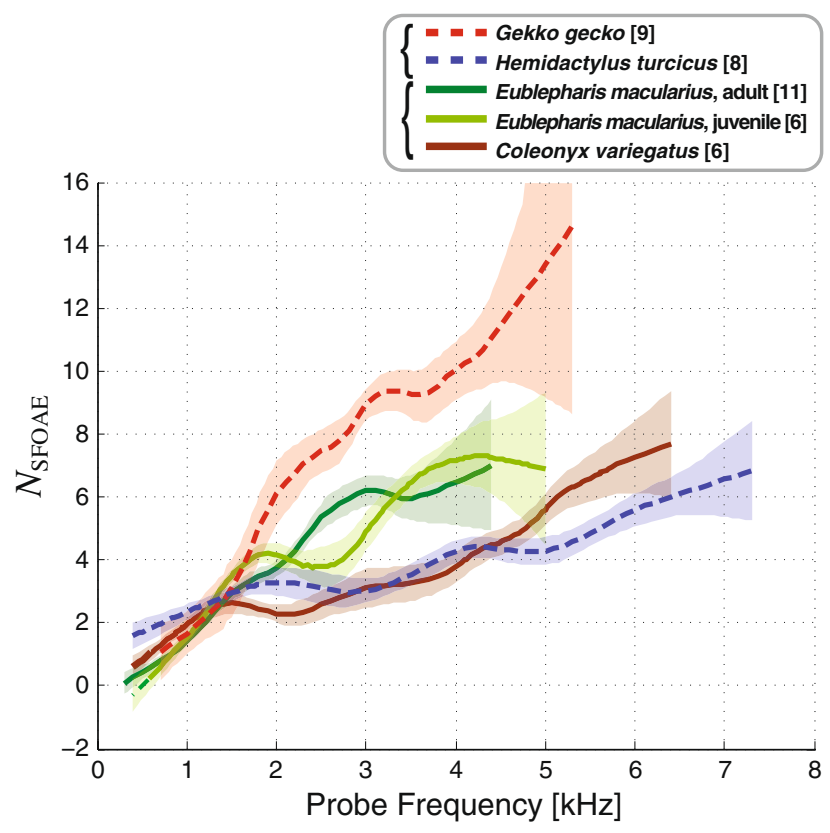

FIG. 4. Comparison of SFOAE phase-gradient delays (expressed in \# of stimulus cycles, $N_{\mathrm{SFOAE}}$ ) across gecko species (see caption to Fig. 1). Same data as shown in previous figures. Species within similar subfamily pairings are grouped by line style: Gekkoninaedashed lines, Eublepharinae-solid lines. In figure legend, the number in brackets indicates number of unique ears included in the trend. Brackets on left of legend indicate same subfamily pairings as in Table 1. Shaded regions indicate associated 95\% confidence interval. Probe level, $L_{p}=20 \mathrm{~dB}$ SPL.

bins (see Methods). Results are summarized in Table 3.

Several features of the emission magnitudes are apparent. Firstly, each ear exhibits a plateau in the response magnitude punctuated by a unique set of peaks and valleys that are stable provided body temperature is kept constant. Secondly, magnitudes at moderate frequencies $(1-3 \mathrm{kHz})$ are similar for similarly sized species across subfamilies (e.g., Hemidactylus and Coleonyx levels were similar). However, within a given pair, magnitudes are slightly higher for the larger species. Thirdly, emissions extend out to higher frequencies $(>3-4 \mathrm{kHz})$ for the smaller species (right column in Figs. 1 and 2) before falling off towards the noise floor. Fourthly, at the lowest frequencies where emissions are reliably measured above the noise floor $(\sim 0.5-1 \mathrm{kHz})$, magnitudes are similar for Eublepharis and Coleonyx, but slightly larger in Gekko relative to Hemidactylus. Lastly, magnitudes are similar between adult and juvenile Eublepharis, though they extend out towards slightly higher frequencies in the juveniles before falling off towards the noise floor.

Emissions from all ears exhibit significant phase accumulation as the stimulus tone is swept (Figs. 1, 2, and 3), indicative of delays on the order of milliseconds (Table 3$)$. The time delays $\left(\tau_{\mathrm{OAE}}\right)$ range from
1 to $3 \mathrm{~ms}$ for all species. For all species except Hemidactylus, $\tau_{\mathrm{OAE}}$ initially increases with frequency, reaching a plateau and then typically decreases. For Hemidactylus, $\tau_{\mathrm{OAE}}$ monotonically decreases with frequency from $\sim 2.6 \mathrm{~ms}$ at $0.4 \mathrm{kHz}$ to $\sim 0.9 \mathrm{~ms}$ at $7.1 \mathrm{kHz}$. Values are compiled together across species in Figure 4 , showing the delay plotted non-dimensionally in number of stimulus cycles $\left(N_{\mathrm{SFOAE}}\right)$. While $\tau_{\mathrm{OAE}}$ and $N_{\mathrm{SFOAE}}$ are directly proportional (Eq. 1), the latter provides a more direct value for comparing to estimates of tuning such as the quality factor $(Q)$. From Figure 4, it is apparent that for the smaller species within a given pair (as indicated by similar line styles), $N_{\mathrm{SFOAE}}$ values are relatively smaller. Furthermore, values are fairly similar between juvenile and adult Eublepharis, though some differences exist around $2-3 \mathrm{kHz}$. Additionally, there is little difference apparent below $\sim 1.2 \mathrm{kHz}$, except for Hemidactylus, which maintains relatively high $N_{\mathrm{SFOAE}}$ values. The upward $N_{\mathrm{SFOAE}}$ trends in Figure 4 indicate that the delay (in number of stimulus cycles) increases with frequency, similarly to ANF tuning estimates (Eatock et al. 1982; Sams-Dodd and Capranica 1994; Manley et al. 1999).

Despite the tight interaural coupling in lizards (see 'Discussion'), emissions from both ears within an individual gecko appear independent of one another: Each ear produces its own unique set of magnitude peaks and valleys with respect to frequency. Upon visual inspection, comparison of SFOAE response curves (magnitude and phase) in both ears of one individual are typically no more similar or different than when comparing across two unique individuals. This observed independence is consistent with a previous report for skink SOAEs (Köppl and Manley 1993b) and provides justification for pooling data together when computing the loess trends. While data from three distinct ears (from as many different individuals) alone can be sufficient to compute the $N_{\mathrm{SFOAE}}$ trend, including as many unique ears as possible captures the most robust fit (Bergevin et al. 2010b) and associated confidence interval.

Although not systematically reported here, the results indicated above for SFOAEs were fairly similar to those observed for other emission types. For example, some degree of SOAE activity was present in most individual Hemidactylus and Coleonyx tested. Once in the steady-state warm temperature condition, SOAE peaks were routinely seen for Hemidactylus upwards of $\sim 6-7 \mathrm{kHz}$ and for Coleonyx up to $\sim 5-$ $6 \mathrm{kHz}$. Overall, SOAE activity appeared more robust in Gekko and Eublepharis relative to Hemidactylus and Coleonyx respectively, and furthermore activity was greater in Hemidactylus relative to Coleonyx (similar to greater SOAE activity in Gekko compared with Eublepharis). SOAEs from juvenile Eublepharis have similar 
frequency distributions to those previously reported for adults (Manley et al. 1996), though appear to extend out towards slightly higher frequencies, similar to the SFOAE results indicated here. Furthermore, distortion product emissions (DPOAEs), evoked using equal-level primaries at $45 \mathrm{~dB}$ SPL at $f_{2} / f_{1}$ ratios of $1.07,1.22$, and 1.35 , also exhibit relatively larger magnitudes at higher frequencies in the smaller species.

Given that lizards are ectothermic and that body temperature influences OAEs (e.g., van Dijk et al. (1989); Manley et al. (1996); Bergevin et al. (2010a)), it is important to briefly consider how temperature could affect the present data. Emissions extend out towards higher frequencies with increasing body temperatures. A similar effect is also seen at the level of the ANF: Characteristic frequencies shift upwards with temperature for both Gekko (Eatock and Manley 1982) and spectacled caiman (Caiman crocodilus) (Smolders and Klinke 1984), though there is little effect upon tuning sharpness. Furthermore, based upon electrophysiological measures, there may be an 'optimal' temperature for the response from the ear (Werner 1972, 1976). All lizards in this study were kept stable at the same body temperature of approximately $32-33^{\circ} \mathrm{C}$. While the criteria for establishing such an optimal temperature is subjective and could vary across species, detailed consideration of such is beyond the scope of the present paper. It is suggested here that variations in such would be small across the species within a pair (given broadly similar ecological habitats) and not have a significant impact upon the present results.

\section{DISCUSSION}

The approach here has been a comparative one: examining auditory function and attempting to simplify interpretation by using phylogenetically matched pairings. The data provide answers to the questions originally posed: 1) ears with fewer hair cells/shorter papillae exhibit slightly smaller SFOAE magnitudes $(<3-4 \mathrm{kHz})$ and significantly shorter delays $(>1.5 \mathrm{kHz}), 2)$ emissions extend to higher frequencies in the smaller species, and 3) there is relatively little difference in SFOAEs between juvenile and adult Eublepharis (in contrast to differences between adult Eublepharis and Coleonyx). The discussion will now attempt to provide some rationale for these observations.

\section{Improved high-frequency sensitivity in smaller lizards?}

The extension of emissions to higher frequencies in the smaller species of a pair (Table 3) suggests an extended range of active hearing and subsequently, increased high-frequency sensitivity. While the presence (or absence) of OAEs may not be a definitive indicator of whether a species is sensitive to a particular frequency region, various lines of evidence support such a notion. Firstly, this high-frequency extension is consistent with the anatomical observation of the thinner fundus in the smaller species (i.e., reduced mass of BM load) (Wever 1978), which could shift the resonant frequency of the papilla upwards. ${ }^{1}$ Secondly, the apical portion of the papilla (sensitive to higher frequencies) appears disproportionally larger in the smaller species (Wever 1978). Thirdly, the extension towards higher frequencies appears consistent with a comparison of tympanic membrane velocity response curves in phylogentically matched gecko pairs (Werner et al. 2002). Fourthly, SOAEs and low-level SFOAEs rapidly fall off towards the noise floor above the highest ANF characteristic frequencies in Gekko (Manley et al. 1996; Bergevin and Shera 2010). Fifthly, SOAE suppression characteristics and SFOAE delays have been shown to correlate well to ANF tuning (Köppl and Manley 1994; Manley et al. 1996; Bergevin and Shera 2010). Lastly, ABR audiograms for Anolis carolinensis extend out to higher frequencies relative to Gekko (Brittain-Powell et al. 2010), consistent with OAE magnitudes (Manley and Gallo 1997; Bergevin et al. 2010b).

The observation regarding size and high-frequency emissions is also consistent with other lizard groups: a previous study made the general observation across species that the larger the lizard, the lower the frequency at which emissions fell off to the noise floor (Bergevin et al. 2010b). One notable exception was Callisaurus draconoides, a medium-sized member of the Phrynosomatidae family, whose emissions fell off at relatively low frequencies. This is likely due to poor middle-ear transmission (Bergevin et al. 2010b), indicating other factors besides size bear consideration. It was recently shown that papilla length does not correlate well to the high-frequency limit of hearing (Manley 2010) and that other morphological aspects likely play a role (e.g., TM morphology, subdivision of papilla into mirrored regions). Hence, an underlying motivation for the present study: minimize potentially confounding differences by comparing

\footnotetext{
While the papilla is not tuned in the traditional sense of a longitudinal tonotopic variation, its response is in fact tuned. Reports looking at BM motion in reptiles indicate that papilla motion tends to be band-pass in nature (e.g., similar to a secondorder filter, such as a harmonic oscillator or an RLC circuit) (Peake and Ling 1980; Rosowski et al. 1985; Manley et al. 1988; O'Neill and Bearden 1995; Aranyosi and Freeman 2005; Chiappe 2006). In these studies, the BM of larger lizards (skink and tokay gecko) was tuned to a lower frequency relative to a smaller one (alligator lizards).
} 
within closely matched pairs. Two salient functional rationales are now proposed to help explain why the smaller species in the pair appears more sensitive at higher frequencies: sound localization and vocalization.

On a basic level, binaural information important for azimuthal sound localization depends upon interaural spacing. Phase differences (or interaural time differences (ITDs)) across the two ears provide an important cue for discrimination, in addition to interaural level differences that stem from diffraction about the head (e.g., acoustic shadowing) and filtering by the external ear (see Popper and Fay (2005)). Animals with smaller interaural spacing can be at a disadvantage for ITD-based sound localization relative to larger species, since ITDs become negligible at higher frequencies. Increased high-frequency sensitivity can however compensate for such a disadvantage (Heffner and Heffner 2008). While ITD-coding in mammals typically requires precise neural timing mechanisms, the strong interaural coupling in lizards and frogs allows their middle ear to essentially act as a pressure difference receiver (Christensen-Dalsgaard and Manley 2005; Ho and Narins 2006; ChristensenDalsgaard and Manley 2008). Because of the coupling of the two eardrums, azimuthal location of the sound source can lead to a large difference in the tympanic response of the two ears. Sound location is thereby potentially encoded as a level difference between the two, thus obviating the need for critical spike-by-spike timing precision. Such a pressure difference model can readily be described via a simple lumped-element approach (Fletcher and Thwaites 1979; Fletcher 1992), though extension to higher frequencies $(\geq 2$ $3 \mathrm{kHz}$ ) may be difficult due to the acoustic wavelength becoming comparable in size to the physical dimensions of the auditory structures (Fletcher 1992). Nonetheless, physiological measurements indicate that the "frequency at peak directionality" is generally higher for those lizards with smaller interaural spacing (Christensen-Dalsgaard and Manley 2005), independent of modeling considerations. Thus we might expect such a trend in the lizard pairings here: Improved azimuthal sound localization accompanies greater high-frequency sensitivity in the smaller species, thereby offsetting narrower interaural separation.

A second consideration concerns the vocalizations of geckos, who are well known to be highly communicative (unlike most other lizards) and exhibit spectrally rich utterances. It stands to reason that such vocalizations are ecologically important and thus tied back to the performance of the inner ear. The frequency range over which OAEs occur matches well to that of the gecko's vocalizations (Marcinelli 1977; Bergevin et al. 2010b). In terms of production apparatus, there can be significant morphological variation in vocal tract across gecko species (Russell et al. 2000). Differences in body size of the species examined here (Table 1) may be expected to have corresponding changes at the level of the vocal tract. For example, vocalizations from the smaller species may shift towards higher frequencies due to decreased glottal mass or increased stiffness. In one species of gecko (Ptenopus garrulus garrulus), the "dominant call frequency" has been reported to decrease with increasing SVL (Hibbitts et al. 2007). Although there are many different types of utterances with varying frequency content (e.g., mating versus territorial calls, single versus multiple "chirps"), spectrograms suggest that smaller gecko species may have energy extending out to higher frequencies than larger species (Marcinelli 1977; Frankenberg 1982). An inverse relationship between body size and the upper limit of relevant frequency content in vocalizations thus appears congruous with the extended frequency range of emissions in the smaller lizards.

\section{Source of SFOAE delay?}

One salient focus of the present study is the SFOAE steady-state phase-gradient delays (e.g., Table 3, Fig. 4), which are relatively large: $\tau_{\mathrm{OAE}}$ is several milliseconds, or equivalently, several stimulus cycles (when expressed as $N_{\mathrm{SFOAE}}$ ). What is the source of these long delays? As noted previously, it has been suggested that SFOAE delays provide a means to noninvasively estimate the frequency selectivity of the inner ear. However, additional delay sources besides those associated with the tuning of the underlying filters (e.g., middle-ear transmission) will undoubtedly affect emission phase and thereby contribute to SFOAE delays. The amphibian ear provides a case in point: long SFOAE delays with relatively broad ANF tuning (Meenderink and Narins 2006; Bergevin et al. 2008). A recent report for the frog (van Dijk et al. 2010) suggests that an additional delay of as much as $1 \mathrm{~ms}$ arises from the tympanic membrane response. It is presently unclear if such a large delay may be uniquely characteristic of amphibious species. Nonetheless, it is worthwhile to consider how additional delay sources could contribute to the interpretation of the present SFOAE data.

One possible delay source could be due to energy propagation to/from the emission generation site inside the inner ear. Relevantly, there is presently significant debate for the mammalian cochlea about differences between the forward (stimuli going to generation site) and reverse paths (emission traveling from generation site back towards stapes) and how the associated delays could be quite different (e.g., Siegel 
et al. (2005); Ren et al. (2006); Dong and Olson (2008); Shera et al. (2008); Meenderink and van der Heijden (2010)). The relative simplicity of the lizard ear avoids such a complication: given the apparent lack of BM traveling waves in geckos, energy associated with OAE generation likely travels via fast compressional waves and differences across the species here will thus be negligible. For example, assuming the speed of sound in water to be $\sim 1,500 \mathrm{~m} / \mathrm{s}$, the delay associated with an extra $2 \mathrm{~mm}$ distance (to and from the longest papilla difference listed in Table 1) would be about $0.001 \mathrm{~ms}$, three orders of magnitude smaller than SFOAE delays observed (Table 3 ).

The middle ear (ME) is another possible delay source, as sound energy propagates from outside the tympanic membrane to inside the stapeidal footplate (and additionally in reverse direction for OAEs). Transmission delays of the mammalian ME are relatively short, typically on the order of tens of microseconds (Guinan and Peake 1967; Dong and Olson 2006). The ME is relatively simpler in lizards: only one ossicle (columella), as opposed to three in the mammalian ear. Although there is apparently no definitive measure of ME delay in the lizard at present, previous reports suggest that forward ME delays in several reptile species are relatively small (Rosowski et al. 1985; Wilson et al. 1985; Manley et al. 1988; Werner et al. 1998) and are (at least) roughly an order of magnitude shorter than SFOAE delays. Furthermore, ME delays between juvenile and adult Eublepharis appear fairly similar (Werner et al. 1998), indicating size of the middle ear is not a critical factor.

Taken together, the available data suggest that delay sources stemming from middle-/inner-ear transmission are relatively small and/or similar within phylogenetically matched pairs. This conclusion is further reinforced by the similarity between juvenile and adult Eublepharis data (Table 3), indicating that body size alone does not appear to fundamentally determine the associated delays. So what then is the major source of these long SFOAE delays, and how do we interpret differences in such across species? In a nutshell, build-up time of the underlying auditory filters due to their sharp tuning can account for these emission latencies (Smolders and Klinke 1986; Shera et al. 2002; Moleti and Sisto 2003). Furthermore, a model for such inspired by the lizard ear appears fairly robust to the underlying filter assumptions and suggests broad applicability across species (Bergevin and Shera 2010). As such, inferring differences in tuning across species as shown in Figure 4 suggests that the smaller species within a pair has relatively broader tuning. Is such a conclusion consistent with the morphology and other SFOAE properties?

\section{Fewer sensory cells=broader tuning?}

First, it is important to consider how the frequency range of audition compares across species within a pair. Since the papilla has an exponential tonotopic organization (Manley et al. 1999) whose tuning can be characterized by an associated mapping constant (papillar length per octave spanned), species with relatively broader tuning (for similar frequency ranges spanned) would have a smaller associated mapping constant. ${ }^{2}$ As noted in a previous section, SFOAE and anatomical data suggest greater sensitivity to higher frequencies in Hemidactylus and Coleonyx relative to Gekko and Eublepharis, respectively. Though the lower end of the frequency range could also be different, such a notion is presently hard to address given that emissions are difficult to measure below $\sim 0.4-0.5 \mathrm{kHz}$ and that $\mathrm{OAE}$ generation mechanisms could be different for the lower frequency portion of the papilla. But given the data in hand, it seems reasonable to surmise that the smaller lizards have similar (if not greater) frequency ranges relative to the larger species. Given the shorter papilla length (Table 1), this would mean a relatively smaller mapping constant (i.e., broader tuning).

Next, we consider the dissimilar number of hair cells between species within a pair (Table 1). To first order, if the two related lizard species have a dissimilar number of hair cells, it may be expected that the tuning would be broader in the species with fewer hair cells since there will be less filters to cover the frequency range spanned. The handicap of fewer hair cells could be offset by several possibilities. Firstly, differences in the frequency range of audition: spanning fewer octaves for a shorter papilla could lead to a similar mapping constant as the larger papilla. However, as suggested in the previous paragraph, the frequency ranges appear roughly similar between the two, reinforcing the notion of broader tuning in the smaller species. Secondly, it is possible

\footnotetext{
$\overline{2}$ To first order, the Gekko tonotopic map seems well approximated by the function $f(x)=f_{\max } e^{-x / \ell}$, where $x$ is position along the papilla length (from the apical end), fmax is the highest center frequency $(\approx 5 \mathrm{kHz})$, and $\ell$ is the associated space constant $(\mathrm{mm})$. According to the data from Manley et al. (1999), Bergevin and Shera (2010) estimated the space constant for Gekko (the only gecko species in which ANF tracing measurements have been reported to our knowledge) as $\ell=0.5 \mathrm{~mm}$. Slightly different is the mapping constant $(\equiv \beta)$ (mm/oct.) (Manley et al. 1999). For the simple exponential map noted above, it is relatively straightforward to show the two constants are simply proportional: $\beta=\ell \ln (2)$. Thus there is a slight discrepancy in the estimated mapping constants for Gekko between Manley et al. (1999) and Bergevin and Shera (2010) (0.4 and $0.35 \mathrm{~mm} /$ oct. respectively).
} 
that there could be differences in filter density (i.e., \# of hair cells/octave). The density would likely be critically tied to morphological properties (Miller 1973a, b; Wever 1978) such as the spacing between adjacent hair cells (which appears relatively uniform across species) and number of coupled cells per sallet. A rough approximation of filter density can be inferred from Table 1 by dividing total cell count by papilla length, suggesting fewer cells per unit length in the smaller species (consistent with broadened tuning). However, a disproportionately larger salletal region in the smaller species (Wever 1978) could offset such a simplified consideration.

To summarize, several lines of evidence have thus been raised: shorter SFOAE delays above $\sim 1.3 \mathrm{kHz}$ (consistent with the frequency range of the salletal region) for the smaller species within a matchedpair, theoretical considerations relating these delays to tuning of the auditory filters in the gecko inner ear (Bergevin and Shera 2010), ${ }^{3}$ and simple morphological considerations (e.g., fewer salletal hair cells in smaller species). These various observations are congruous and lead to the same conclusion: broader tuning above $\sim 1.3 \mathrm{kHz}$ in the smaller species within a given pair. Furthermore, this notion appears consistent with a previous study (Bergevin et al. 2010b) when comparing tuning estimates within a phylogentically matched group: in the family Phrynosomatidae (small papillar length, lack of TM over majority of papilla), species with more hair cells generally had higher $N_{\mathrm{SFOAE}}$ values, suggestive of sharper tuning.

\section{Juvenile versus adult}

A previous study of study examining age-dependence of auditory function in Eublepharis indicated sensitivity differences (Werner et al. 1998), adults appearing slightly more sensitive than juveniles. Mechanisms for such an age-related difference might vary across gecko species, but appear partially attributable to changes in middle-ear function related to body size/ length (Werner et al. 2002, 2008). Previous anatomical studies (Miller 1985; Gehr and Werner 2005)

\footnotetext{
3 The results of that model were not sensitive to the number of "bundles" $(n)$, provided that this value was above some minimal number $(\sim 20)$. This lack of sensitivity apparently arises because it is the explicit dependence of $Q$ upon $f$ that matters. The means of extracting such a relationship from ANF data (e.g., Manley et al. (1999)) as described in Bergevin and Shera (2010) made no explicit assumption about $n$, thus its apparent irrelevance. Put another way, the dependence upon $n$ was already implicitly taken into account via the ANF data that formed a key model input parameter (i.e., $Q(f)$ ). Thus, the apparent paradox does not in fact exist and the theory is ultimately consistent with experiment: the number of sensory cells does indeed factor in to tuning sharpness.
}

suggest the lizard inner ear is fairly well-formed at birth, though juvenile Eublepharis and adult Coleonyx appear remarkably similar in appearance and external size. Given the relative similarity in emissions between juvenile and adult Eublepharis (and their contrast to Coleonyx data), the present results suggest that OAE properties derive primarily from characteristics of the inner ear. Despite the overall similarity, several differences in SFOAEs between the two Eublepharis age groups were noted. Emissions extend out to slightly lower frequencies in adults (Table 3), which could be in part due to some degree of presbycusis and/or middle-ear transmission (Werner et al. 1998, 2002, 2008). Also, differences in $N_{\mathrm{SFOAE}}$ are apparent from $2-3 \mathrm{kHz}$ (Fig. 4), which may parallel age-related effects observed in human DPOAE phase-gradient delays (Abdala and Dhar 2010).

\section{Predictions for other measures}

Given the interest in extending interpretation of $N_{\mathrm{SFOAE}}$ to tuning measures in mammalian species (Shera et al. 2002, 2010), where BM wave delays potentially play an additional complicating role, it could prove instructive to use other measurement modalities to further explore the conclusions drawn here. Several specific testable hypotheses are noted towards this end. First, the present results suggest that ANF tracing measurements that determine the tonotopic map (e.g., Manley et al. (1999)) would find lower mapping constants for the smaller species, consistent with broader tuning. Similarly, tuning ratios (e.g., ratio of ANF-derived $Q$ to $N_{\mathrm{SFOAE}}$, Shera et al. (2010)) are expected to be relatively invariant across species. Second, ABR audiograms (e.g., Brittain-Powell et al. (2010)) for Hemidactylus and Coleonyx would be predicted to exhibit lower thresholds at higher frequencies than those of Gekko and Eublepharis respectively, though differences in ANF population size/innervation density need to be considered. Third, ABR tuning curves would be expected to be broader in Hemidactylus and Coleonyx relative to Gekko and Eublepharis, respectively. This broader tuning may also be apparent via shorter ABR wave 1 latencies in the smaller species. Fourth, a systematic comparison of low-level SFOAEs and DPOAE suppression via a third tone (e.g., Brown and Kemp (1984); Köppl and Manley (1993a); Kössl et al. (1996); Martin et al. (1998)) should help reconcile distinctions between tuning as measured via excitation versus suppression (e.g., Lineton and Wildgoose (2009)). Lastly, the present conclusions are likely not limited to geckos: presumably, any lizard pairings analogous to the ones chosen here should demonstrate qualitatively similar relation- 
ships. Furthermore, extending beyond lizards to comparatively examine SFOAEs in phylogentically matched groups of mammals or other non-mammals such as birds (e.g., Gleich and Langemann (2010)) could prove valuable.

\section{Summary}

The comparative approach taken here describes emission properties that can be traced down to known quantitative morphological details at the level of the inner ear. The results of the study suggest the following conclusions when comparing species within gecko subfamilies: (1) broader tuning in papillae with fewer hair cells, (2) increased highfrequency sensitivity in smaller species with shorter interaural separation, and (3) differences in OAE properties with age within a given species are relatively small despite body size differences, consistent with notion that inner ear function is adult like within at least $1-2$ weeks post-hatching. Taken together, the present results indicate that size does indeed matter for emission generation, but primarily at the level of the inner ear. If the inner ear is fairly well-formed, then there is little difference in emission properties even with large differences in overall body size within a given species. However, when comparing across different species, (adult) body size is a factor since it appears intimately tied to the dimensions of the inner ear.

\section{ACKNOWLEDGMENTS}

Support and encouragement from K. Bonine and D. Velenovsky were invaluable for the completion of this study. Veterinary support was provided by T. Cioni, J. Jarchow and N. McMullen. K. Baker, M. deMarco and the Arizona Game and Fish Department helped in procuring animals. Input from A.P. Russell on gecko vocalizations is appreciated. Suggestions regarding computation of the loess trends (including bootstrapping) from C. Shera are appreciated. Comments from S. Meenderink, E. Olson, J. Saunders, C. Shera, and the anonymous reviewers helped improve upon an earlier draft. Financial support came from the Howard Hughes Medical Institute (52003749), National Science Foundation, Division of Mathematical Sciences (0602173), and the National Institutes of Health (R01 DC3687).

\section{REFERENCES}

AbDala C, Dhar S (2010) Distortion product otoacoustic emission phase and component analysis in human newborns. J Acoust Soc Am 127:316-325

Aranyosi A, Freeman D (2005) Two modes of motion of the alligator lizard cochlea: measurements and model predictions. J Acoust Soc Am 118:1585-1592
Bergevin C, Shera CA (2010) Coherent reflection without traveling waves: on the origin of long-latency otoacoustic emissions in the gecko. J Acoust Soc Am 127:2398-2409

Bergevin C, Freeman D, Saunders J, Shera C (2008) Otoacoustic emissions in humans, birds, lizards, and frogs: evidence for multiple generation mechanisms. J Comp Physiol A 194:665-683

Bergevin C, Velenovsky D, Bonine K (2010A) Otoacoustic emission temperature dependence across the lacertilia. In: Abstracts of the thirty-third midwinter research meeting. Association for Research in Otolaryngology, Anaheim

Bergevin C, Velenovsky D, Bonine K (2010B) Tectorial membrane morphological variation: effects upon stimulus frequency otoacoustic emissions. Biophys J 99:1064-1072

Brittain-Powell E, Christensen-Dalsgaard J, Tang Y, Carr C, Dooling $\mathrm{R}$ (2010) The auditory brainstem response in two lizard species. J Acoust Soc Am 128:787-794

Brown A, Kemp D (1984) Suppressibility of the 2f1-f2 stimulated acoustic emissions in gerbil and man. Hear Res 13:29-37

Campbell H (1969) The effects of temperature on the auditory sensitivity of lizards. Physiol Zool 42:183-210

CHIAPPE E (2006) Functional Differentiation of hair cells in a lizard papilla unveils a principle in the evolution of amniote cochleae. Ph.D. thesis, Rockefeller University

Chiappe M, Kozlov A, Hudspeth A (2007) The structural and functional differentiation of hair cells in a lizard's basilar papilla suggests an operational principle of amniote cochleas. J Neurosci 27:11978-11985

Christensen-Dalsgaard J, Manley G (2005) Directionality of the lizard ear. J Exp Biol 208:1209-1217

Christensen-Dalsgaard J, Manley G (2008) Acoustic coupling of lizard eardrums. J Assoc Res Otolaryngol 9:407-416

Cleveland W (1993) Visualizing Data. Hobart Press, Summit, NJ

Dong W, Olson E (2006) Middle ear forward and reverse transmission in gerbil. J Neurophysiol 95:2591-2961

Dong W, Olson E (2008) Supporting evidence for reverse cochlear traveling waves. J Acoust Soc Am 123:222-240

Eatock R, Manley G (1982) Auditory nerve fiber activity in the gecko. II. temperature effect on tuning. J Comp Physiol A 142:219-226

Eatock R, Manley G, Pawson L (1982) Auditory nerve fiber activity in the gecko. I. implications for cochlear processing. J Comp Physiol A 142:203-218

Fletcher N (1992) Acoustics systems in biology. Oxford University Press, New York

Fletcher N, Thwaites S (1979) Physical models for the analysis of acoustical systems in biology. Q Rev Biophys 12:25-65

FRANKENBERG E (1982) Vocal behavior of the mediterranean house gecko, hemidactylus turcicus. Copeia 1982:770-775

Ganeshina O, Vorobyev M (2009) A contractile cochlear frame is a common feature of the hearing organs in Gekkota (sauria, Squamata): a comparative study. Brain Behav Evol 74:87-101

GeHr D, Werner Y (2005) Age effects and size effects in the ears of gekkonomorph lizards: inner ear. Hear Res 200:38-50

Gelfand M, Piro O, Magnasco M, Hudspeth A (2010) Interactions between hair cells shape spontaneous otoacoustic emissions in a model of the Tokay geckos cochlea. PLoS ONE 5

Gleich O, Langemann U (2010) Auditory capabilities of birds in relation to the structural diversity of the basilar papilla. Hear Res. doi:10.1016/j.heares.2010.01.009

Guinan J, Peake W (1967) Middle-ear characteristics of anesthetized cats. J Acoust Soc Am 41:1237-1261

Heffner H, Heffner R (2008) High-frequency hearing. In: Dallos P, Oertel D, Hoy R (eds) Audition, handbook of the senses. Elsevier, London, pp 55-60

Hibbitts T, Whiting M, Stuart-Fox D (2007) Shouting the odds: vocalization signals status in a lizard. Behav Ecol Sociobiol 61:1169-1176 
Ho C, NARINS P (2006) Directionality of the pressure-difference receiver ears in the northern leopard frog, Rana pipiens pipiens. $\mathrm{J}$ Comp Physiol A 192:417-429

Köppl C, AuthiER S (1995) Quantitative anatomical basis for a model of micromechanical frequency tuning in the tokay gecko, Gekko gecko. Hear Res 82:14-25

Köppl C, MANLey G (1993A) Distortion-product otoacoustic emissions in the bobtail lizard. II: suppression tuning characteristics. J Acoust Soc Am 93:2834-2844

Köppl C, Manley G (1993B) Spontaneous otoacoustic emissions in the bobtail lizard. I: general characteristics. Hear Res 71:157-169

Köppl C, Manley G (1994) Spontaneous otoacoustic emissions in the bobtail lizard. II: interactions with external tones. Hear Res 72:159-170

Kössl M, Frank G, Burda H, Müller M (1996) Acoustic distortion products from the cochlea of the blind Arican mole rat, cryptomys spec. J Comp Physiol A 178:427-434

Liberman M (1990) Effects of chronic cochlear de-efferentation on auditory-nerve response. Hear Res 49:209-224

Lineton B, Wildgoose C (2009) Comparing two proposed measures of cochlear mechanical filter bandwidth based on stimulus frequency otoacoustic emissions. J Acoust Soc Am 125:1558-1566

ManLEY G (1997) Diversity in hearing-organ structure and the characteristics of spontaneous otoacoustic emissions in lizards. In: Lewis E, Long G, Lyon R, Narins P, Steele C (eds) Diversity in auditory mechanics. World Scientific, Singapore, pp 32-38

ManLEY G (2000) Cochlear mechanisms from a phylogenetic viewpoint. Proc Natl Acad Sci USA 97:11736-11743

MANLEY G (2002) Evolution of structure and function of the hearing organ of lizards. J Neurobiol 53:202-211

Manley G (2006) Spontaneous otoacoustic emissions from freestanding stereovillar bundles of ten species of lizard with small papillae. Hear Res 212:33-47

MANLEY G (2009) Spontaneous otoacoustic emissions in lizards: a comparison of the skink-like lizard families cordylidae and gerrhosauridae. Hear Res 255:58-66

MANLEy G (2010) Lizard auditory papillae: an evolutionary kaleidoscope. Hear Res. doi:10.1016/j.heares.2010.01.009

Manley G, Gallo L (1997) Otoacoustic emissions, hair cells, and myosin motors. J Acoust Soc Am 102:1049-1055

Manley G, Kraus E (2010) Exceptional high-frequency hearing and matched vocalizations in australian pygopod geckos. J Exp Biol 213:1876-1885

Manley G, Yates G, Köprl C (1988) Auditory peripheral tuning: evidence for a simple resonance phenomenon in the lizard tiliqua. Hear Res 33:181-190

Manley G, Gallo L, Köppl C (1996) Spontaneous otoacoustic emissions in two gecko species, Gekko gecko and Eublepharis macularius. J Acoust Soc Am 99:1588-1603

Manley G, Köppl C, Sneary M (1999) Reversed tonotopic map of the basilar papilla in Gekko gecko. Hear Res 131:107-116

Marcinelli D (1977) Acoustic and visual display behavior in Gekkonid lizards. Am Zool 17:251-260

Martin G, Jassir D, Stagner B, Whitehead M, Lonsburi-Martin B (1998) Locus of generation for the 2f1-f2 vs 2f2-f1 distortionproduct otoacoustic emissions in normal-hearing humans revealed by suppression tuning, onset latencies, and amplitude correlations. J Acoust Soc Am 103:1957-1971

MeEnderink S, Narins P (2006) Stimulus frequency otoacoustic emissions in the northern leopard frog, Rana pipiens pipiens: implications for inner ear mechanics. Hear Res 220:67-75

Meenderink S, van der HejdDen M (2010) Reverse cochlear propagation in the intact cochlea of the gerbil: evidence for slow traveling waves. J Neurosci 103:1448-1455

Miller M (1973A) Scanning electron microscope studies of some lizard basilar papillae. Am J Anat 138:301-330
Miller M (1973в) A scanning electron microscope study of the papilla basilaris of Gekko gecko. Z Zellforsch 136:307-328

Miller M (1985) Quantitative studies of auditory hair cells and nerves in lizards. J Comp Neurol 232:1-24

Moleti A, Sisto R (2003) Objective estimates of cochlear tuning by otoacoustic emission analysis. J Acoust Soc Am 113:423-429

O’Neill M, Bearden A (1995) Laser-feedback measurements of turtle basilar membrane motion using direct reflection. Hear Res 84:125-138

Peake W, Ling A (1980) Basilar-membrane motion in the alligator lizard: Its relation to tonotopic organization and frequency selectivity. J Acoust Soc Am 67:1736-1745

Popper A, FAY R (EDS) (2005) Sound source localization. Springer, New York

Probst R, Lonsbury-Martin B, Martin G (1991) A review of otoacoustic emissions. J Acoust Soc Am 89:2027-2067

Ren T, He W, Scott M, Nuttall A (2006) Group delay of acoustic emissions in the ear. J Neurophysiol 96:2785-2791

Rosowski J, Peake W, Linch T, Leong R, Weiss T (1985) A model for signal transmission in an ear having hair cells with free-standing sterocilia. II. Macromechanical stage. Hear Res 20:139-155

Russell A, Rittenhouse D, Bauer A (2000) Laryngotracheal morphology of afro-madagascan geckos: a comparative survey. J Morphol 245:241-268

SAms-Dodd F, CAPRANICA R (1994) Representation of acoustic signals in the eighth nerve of the tokay gecko: I. Pure tones. Hear Res 76:16-30

Shera CA, Guinan JJ (1999) Evoked otoacoustic emissions arise by two fundamentally different mechanisms: a taxonomy for mammalian OAEs. J Acoust Soc Am 105:782-798

Shera CA, Guinan JJ (2003) Stimulus-frequency-emission group delay: a test of coherent reflection filtering and a window on cochlear tuning. J Acoust Soc Am 113:2762-2772

Shera C, Guinan J, Oxenham A (2002) Revised estimates of human cochlear tuning from otoacoustic and behavioral measurements. Proc Natl Acad Sci USA 99:3318-3323

Shera C, Tubis A, Talmadge C (2008) Testing coherent reflection in chinchilla: auditory-nerve responses predict stimulus-frequency emissions. J Acoust Soc Am 124:381-395

Shera C, Guinan J, Oxenham A (2010) Otoacoustic estimation of cochlear tuning: validation in the chinchilla. J Assoc Res Otolaryngol 11:343-365

Siegel J, Cerka A, Recio-Spinoso A, Temchin A, van Dijk P, Ruggero M (2005) Delays of stimulus-frequency otoacoustic emissions and cochlear vibrations contradict the theory of coherent reection ltering. J Acoust Soc Am 118:2434-2443

Sisto R, Moleti A, Shera CA (2007) Cochlear reflectivity in transmission-line models and otoacoustic emission characteristic time delays. J Acoust Soc Am 122:3554-3561

Smolders J, Klinke R (1984) Effects of temperature on the properties of primary auditory fibres of the spectacled caiman, Caiman crocodilus (L.). J Comp Physiol A 155:19-30

Smolders J, KLINKE R (1986) Synchronized responses of primary auditory fibre-populations in Caiman crocodilus (L.) to singles tones and clicks. Hear Res 24:89-103

Stewart C, Hudspeth A (2000) Effects of salicylates and aminoglycosides on spontaneous otoacoustic emissions in the tokay gecko. Proc Natl Acad Sci USA 97:454-459

van Dijk P, Wit H, Segenhout J (1989) Spontaneous otoacoustic emissions in the european edible frog (Rana esculenta): spectral details and temperature dependence. Hear Res 42:273-282

van Dijk P, Mason M, Schoffelen R, Narins P, Meenderink S (2010) Mechanics of the frog ear. Hear Res. doi:10.1016/j. heares.2010.02.004

Weber E, Werner Y (1977) Vocalizations of two snake-lizards (Reptilia: Sauria: Pygopodidae). Herpetologica 33:353-363 
WERNER Y (1972) Temperature effects on inner-ear sensitivity in six species of iguanid lizards. J Herpetol 6:147-177

WERNER Y (1976) Optimal temperatures for inner-ear performance in gekkonoid lizards. J Exp Zool 195:319-351

Werner Y, Montgomery L, Safford S, Igic P, SAunders J (1998) How body size affects middle-ear structure and function and auditory sensitivity in gekkonoid lizards. J Exp Biol 201:487-502

Werner Y, Igic P, Seifan M, Saunders J (2002) Effects of age and size in the ears of gekkonomorph lizards: middle ear sensitivity. J Exp Biol 205:3215-3223
Werner Y, Montgomery L, Seifan M, Saunders J (2008) Effects of age and size in the ears of gekkotan lizards: auditory sensitivity, its determinants, and new insights into tetrapod middle-ear function. Pflugers Arch 456:951-967

Wever E (1978) The Reptile Ear. Princeton University Press, Princeton

Wever E, Peterson E, Crowley D, Veron J (1964) Further studies of hearing in the gekkonid lizards. Proc Natl Acad Sci USA 51:561-567

Wilson J, Smolders J, KLinke R (1985) Mechanics of the basilar membrane in Caiman crocodilus. Hear Res 18:1-24 